\title{
Blood pressure lowering and stroke prevention: a systematic review and network meta-analysis protocol
}

\author{
Xiao-Ling Zhong ${ }^{1 \#}$, Yi Dong ${ }^{2 \#}$, Wei $\mathrm{Xu}^{3}$, Li Sun ${ }^{1}$, Hui-Fu Wang ${ }^{3}$, Hong-Qi Li ${ }^{2}$, Yu-Yuan Huang ${ }^{2}$, \\ Lan Tan $^{3}$, Qiang Dong ${ }^{4}$, Jin-Tai Yu ${ }^{2}$ \\ ${ }^{1}$ Department of Neurology, Qingdao Central Hospital, Qingdao University, Qingdao 266031, China; ${ }^{2}$ Department of Neurology and Institute \\ of Neurology, WHO Collaborating Center for Research and Training in Neurosciences, Huashan Hospital, Shanghai Medical College, Fudan \\ University, Shanghai 200040, China; ${ }^{3}$ Department of Neurology, Qingdao Municipal Hospital, Qingdao University, Qingdao 266071, China; \\ ${ }^{4}$ Department of Neurology and Institute of Neurology, Huashan Hospital, State Key Laboratory of Medical Neurobiology and MOE Frontiers \\ Center for Brain Science, Fudan University, Shanghai 200040, China \\ Contributions: (I) Conception and design: JT Yu, Q Dong, L Tan; (II) Administrative support: None; (III) Provision of study materials or patients: \\ None; (IV) Collection and assembly of data: XL Zhong, Y Dong, W Xu; (V) Data analysis and interpretation: XL Zhong, Y Dong, W Xu; (VI) \\ Manuscript writing: All authors; (VII) Final approval of manuscript: All authors. \\ \#These authors contributed equally to this work. \\ Correspondence to: Prof. Jin-Tai Yu, MD, PhD. Department of Neurology, Huashan Hospital, Fudan University, 12th Wulumuqi Zhong Road, \\ Shanghai 200040, China. Email: jintai_yu@fudan.edu.cn.
}

\begin{abstract}
Background: Hypertension is a leading cause of stroke and the significance of blood pressure lowering treatment strategies for prevention of stroke has been well established. Despite the established and widespread use of BP lowering drugs, which one is better for stroke prevention is still debated. This study aimed to determine the most effective and safest blood pressure lowering treatments for stroke prevention among various single and combined therapies.

Methods: A systematic search will be performed in PubMed and the Cochrane Library to identify RCTs and meta analyses of different pharmacological interventions for stroke prevention from January 01, 1966 to December 01, 2018. Primary efficacy outcome will be reduction of stroke incidence and safety outcome will be drug-related side effects withdraw. Study quality will be critically appraised based on the sevenpoint tool for assessing risk of bias by Cochrane collaboration. Pairwise meta-analyses and Bayesian network meta-analyses will be performed for all related outcome measures. We will conduct subgroup analyses and sensitivity analyses to assess the robustness of our findings.

Discussion: This network meta-analysis will summarize the direct and indirect evidence aiming to provide a ranking of various blood pressure lowering strategies for prevention of stroke. The results of this metaanalysis may help the physicians in determining the best treatments for their patients in stroke prevention.
\end{abstract}

Trial registration: CRD42018118454.

Keywords: Blood pressure lowering; stroke; meta-analysis

Submitted Jul 26, 2019. Accepted for publication Aug 08, 2019.

doi: $10.21037 /$ atm.2019.08.99

View this article at: http://dx.doi.org/10.21037/atm.2019.08.99

\section{Introduction}

Stroke is a non-communicable neurological disorder affecting people physically, mentally, emotionally, or a combination of the three. In 2017, stroke was the second most frequent cause of death after ischemic heart disease, and caused 6.16 million deaths worldwide (1). Given the high prevalence of stroke, successful prevention in these populations could have important impact on public health. Hypertension is a leading cause of stroke and the 
significance of blood pressure lowering treatments for stroke prevention has been well established. A recently published meta-analysis showed that BP lowering in systolic blood pressure was proportionally associated with a significantly lower risk of stroke (relative risk $=0.73$; 95\% CI: 0.64-0.83) (2). Moreover, the magnitude of BP lowering was linearly associated with the extent of risk reduction for recurrent stroke (3). Largely based on the findings of SPRINT, the 2017 ACC/AHA blood pressure guideline (2017 American College of Cardiology/American Heart Association guideline for the prevention, detection, evaluation, and management of high blood pressure in adults) has recommended intensive BP lowering treatments other than standard BP lowering therapies (4). Despite the established and widespread use of BP lowering drugs, which one is better for stroke prevention is still debated (2,5-8). A meta-analysis published in 2016 demonstrated that Calcium channel blockers were superior to other drugs for the prevention of stroke (2). However, it did not provide head to head comparisons among BP lowering therapies, and failed to do comprehensive subgroup analyses stratified by age, baseline blood pressure, and previous disease history in exploring better treatments for stroke prevention. In addition, it provided no evidence on which BP lowering treatment was more tolerable and safer.

In traditional meta-analysis, only direct comparisons which have been reported in studies are allowed for analysis. However, some blood pressure lowering treatment strategies have never been compared directly in previous trials. This limits identification of the most effective and safest treatment strategy based on direct evidence. Network meta-analyses are an extension of traditional approach enable comparison of multiple treatments in one statistical model (9), and provide a hierarchy on efficacy and safety of these treatments to inform decision making (10).

To our knowledge, no network meta-analysis has been done to explore optimal blood pressure lowering treatments for stroke prevention. Therefore, we plan to perform a traditional and network meta-analyses, comparing the relative efficacy and tolerance of blood pressure lowering treatment strategies for stroke prevention.

\section{Methods}

\section{Overview}

We registered on the international prospective register of systematic review (PROSPERO) to publish our study protocol. The present meta-analysis will follow the PRISMA (preferred reporting items for systematic reviews and meta-analyses) statement (11) and the PRISMA network meta-analysis extension statement (12).

\section{Search strategy and selection criteria}

We will use an existing strategy (13) added with cerebrovascular disorders, stroke, brain infarction, cerebral infarction, brain ischemia, cerebral hemorrhage, or intracranial hemorrhage to identify relevant trials published on PubMed from January 01, 1966 to December 01, 2018. A preliminary search strategy for PubMed is shown in Appendix 1. We will restrict our search to randomized controlled trials, or meta-analyses. We will apply no language restrictions. We will also search the Cochrane Collaboration database. To identify eligible studies, we will manually check the reference lists of the included studies. We will then manually examine whether each trial reported stroke as primary or secondary outcome. Studies will be included for the meta-analysis if they fulfilled the following criteria: (I) randomized controlled trials (RCTs); (II) greater than 1,000 patient-years of follow-up in each study group; (III) trials report stroke as primary or secondary outcome; and (IV) trials use anti-hypertensive drugs for indications other than hypertension. Eligible trials will be categorized into three groups: one or two anti-hypertensive agents against placebo; one or two agents against another; different blood pressure lowering targets. We will not exclude any trials because of the presence of baseline comorbidities.

\section{Data extraction and quality assessment}

The literature search, data extraction, and quality assessment will be done independently by two researchers (XL Zhong and Y Dong). The process of study selection from databases will be shown in Figure 1. Consensus will be achieved through referral to a third reviewer in case of disagreement. Data will be extracted into specially designed excel sheets, including participants' baseline characteristics (age, sex race, mean systolic and diastolic blood pressure levels, history of cardiovascular disease, history of coronary heart disease, stroke, hypertension, diabetes, and atrial fibrillation), achieved blood pressure, mean BP reduction, blood pressure lowering drugs used, follow-up duration, and outcome events. Outcomes of interest will be stroke (fatal or nonfatal, excluding transient ischemic attacks), ischemic stroke, hemorrhagic stroke, fatal or disabling stroke, 


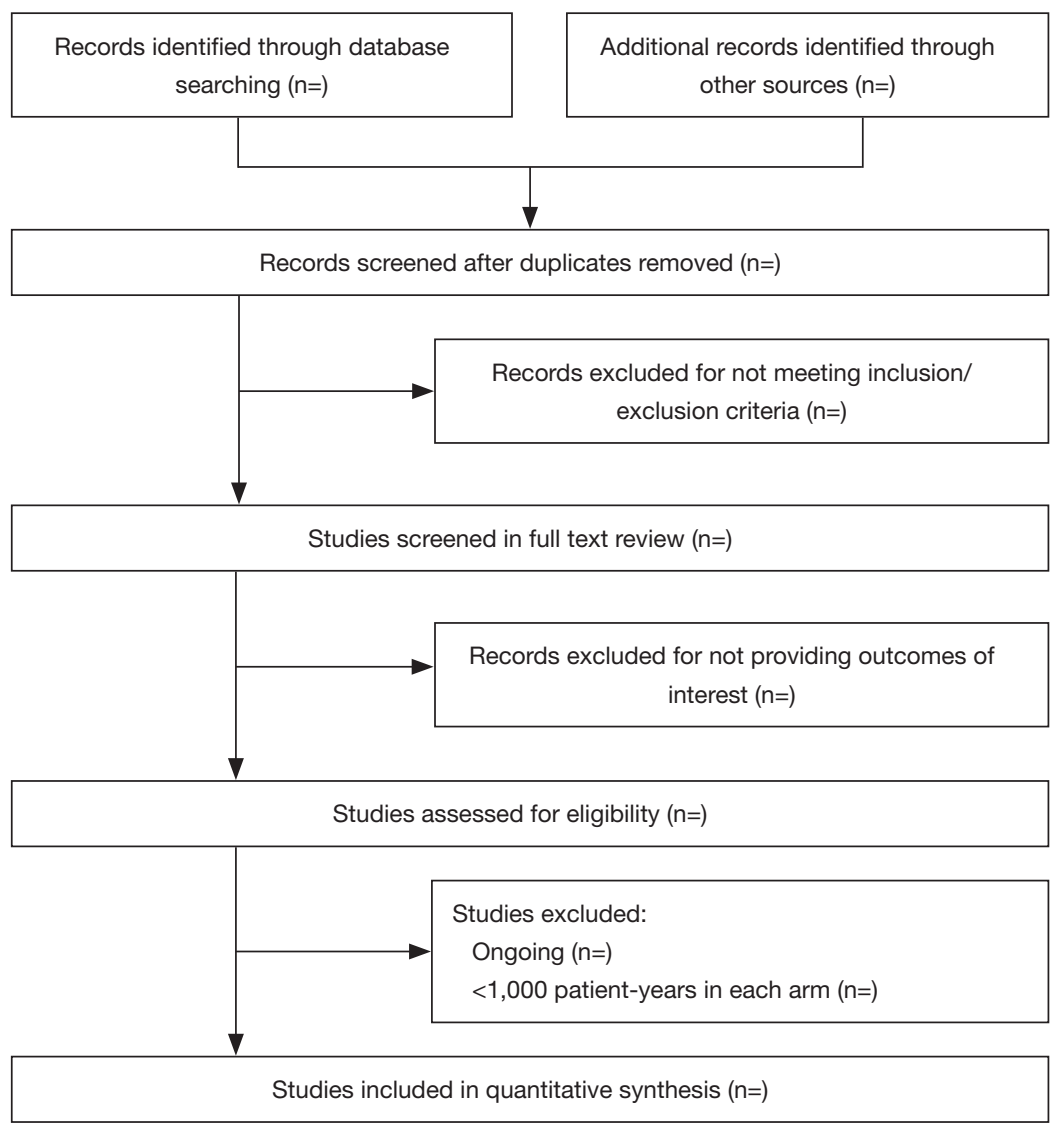

Figure 1 Proposed flow diagram to depict the search process.

cardiovascular death, all cause death. Primary efficacy outcome will be reduction of stroke incidence and safety outcome will be drug-related side effects withdraw. Study quality will be critically appraised based on the seven-point tool for assessing risk of bias by Cochrane collaboration by two researchers who review the literature (14).

\section{Statistical analysis}

To clarify the effects of blood pressure lowering agents on the relative risk of stroke, ischemic stroke, hemorrhagic stroke, fatal or disabling stroke, cardiovascular death and all cause death, we will combine trials of blood pressure lowering agents versus placebo and higher versus lower blood pressure lowering targets and perform traditional meta-analysis. We will calculate relative risks from the number of events and participants for each outcome in each trial and pooled results with Mantel-Haenszel weighted random effects meta-analysis. We will assess the magnitude of statistical heterogeneity among studies using standard
Cochrane chi-square test, the $\mathrm{I}^{2}$ statistic ( $\mathrm{I}^{2}$ values of at least $50 \%$ were considered to represent substantial heterogeneity, while values of at least $75 \%$ indicated considerable heterogeneity (15). We will explore evidence for heterogeneity in estimates of treatment effect attributable to the baseline characteristics of trials by comparing summary results obtain from subsets of studies grouped by age, history of cardiovascular disease, history of stroke, history of diabetes, baseline and achieved blood pressure level. Publication bias will be evaluated both graphically using a funnel plot and with the Egger statistical test for funnel plot asymmetry (16), if at least ten studies are available for each outcome. A leave-one-out sensitivity analysis will be performed by repeating the meta-analysis, each time with one of the included studies omitted, to see whether any one study will be disproportionately large impact on the pooled relative risk.

To clarify the efficacy and safety of different blood pressure lowering drugs for stroke prevention, we will combine the three groups of trials and perform pair-wise 
and network meta-analysis. The outcome measure for efficacy and safety will be stroke and drug-related side effects withdraw, respectively. First, we will perform pairwise meta-analysis with a random effects model to analyze direct treatment comparisons. We will calculate the summary effect sizes as odds ratios, with $95 \%$ confidence intervals. We will assess heterogeneity among studies with the $\mathrm{I}^{2}$ statistic. We will do funnel plots to test publication bias if the comparisons have no less than 10 trials. Second, we will analyze pooled data for all blood pressure lowering treatments with random effects models, within a Bayesian framework in OpenBUGS (17). Models will be computed with Markov chain Monte Carlo simulations, using three chains with over-dispersed initial values, with Gibbs sampling based on 100,000 iterations after a burnin phase of 50,000 iterations. Non-informative or vague priors for the overall mean effect $\left[\theta \sim \mathrm{N}\left(0,100^{2}\right)\right]$ and the between-study standard deviation $[\tau \sim$ uniform $(0,2)]$ will be given (18-20). The mean of the posterior distribution will be reported as the point estimate odds ratio, and the corresponding $95 \%$ credible intervals will be obtained with the 2.5 th and 97.5 th percentiles of the posterior distribution, after adjustment for multiple arm trials. We will test the adequacy of burn-in and convergence (reaching a stable equilibrium distribution) using visual inspection of parameter fluctuation depicted in trace plots and estimating the values of the Brooks-Gelman-Rubin statistic (21). Model fit will be evaluated with the total residual deviance, which indicates good fit, if it approximates the number of data points.

We will assess evidence for consistency in the networks in two ways. First, we will use node-split approach to contrast direct evidence with indirect evidence from the entire network on each node $(20,22,23)$. Second, we will use the design-by-treatment interaction model that provides a single inference, using the $\chi^{2}$ test, about the plausibility of assuming consistency throughout the entire network (24).

The surface under the cumulative ranking curve (SUCRA) and rankograms will be used to provide a hierarchy of the regimens (25). We will also use two-dimensional plots and clustering methods to obtain meaningful groups of the treatments (26). We will assess small study effects with comparison adjusted funnel plot symmetry (26).

To investigate the generalisability of the findings, we will assess the effects of differing trial and participant characteristics on the outcomes in sensitivity analyses by restricting analyses to studies with the following design characteristics: hypertensive participants; excluding heart failure participants; published in 2000 or later; duration of follow-up longer than 3 years. We will perform subgroup analyses according to age (age $\leq 60$ and $>60$ years), history of stroke (no defines as participants with a history of stroke account for less than $5 \%$ of overall participants in a trial, yes defines as all of the participants has a history of stroke in a trial), history of DM (no defines as participants with a history of DM account for less than $5 \%$ of overall participants in a trial, yes defines as all of the participants has a history of DM in a trial), baseline SBP $(\leq 150$ or $>150 \mathrm{mmHg}$ ).

For traditional meta-analyses we will use $\mathrm{R}$ version 3.4.1. For network meta-analyses we will use OpenBUGS 3.2.3 and STATA 14.0.

\section{Discussion}

This network meta-analysis will summarize the direct and indirect evidence aiming to provide a ranking of various blood pressure lowering strategies for prevention of stroke. To the best of our knowledge, this will be the first network meta-analysis performed to explore the efficacy and safety of various antihypertensive treatments for stroke prevention in the whole population. A particular strength of our study is that safety analyses of various blood pressure lowering treatments will be added, we will collect data of drugrelated side effects withdraw in our study to assess the tolerance of different treatments. This has never been done in previous studies. The results of this meta-analysis may help the physicians in determining the best treatments for their patients in stroke prevention.

\section{Acknowledgments}

Funding: This study was supported by grants from the National Natural Science Foundation of China (91849126, 81870915, 81571109), the National Key R\&D Program of China (2018YFC1314700), Shanghai Municipal Science and Technology Major Project (No. 2018SHZDZX01) and ZHANGJIANG LAB, Tianqiao and Chrissy Chen Institute, and the State Key Laboratory of Neurobiology and Frontiers Center for Brain Science of Ministry of Education, Fudan University.

\section{Footnote}

Conflicts of Interest: The authors have no conflicts of interest 
to declare.

Ethical Statement: The authors are accountable for all aspects of the work in ensuring that questions related to the accuracy or integrity of any part of the work are appropriately investigated and resolved. This is a protocol for a systematic review including no confidential personal data and no data on interventions on patients. Therefore, there are no ethical concerns nor informed consent required.

\section{References}

1. Collaborators GBDCoD. Global, regional, and national age-sex-specific mortality for 282 causes of death in 195 countries and territories, 1980-2017: a systematic analysis for the Global Burden of Disease Study 2017. Lancet 2018;392:1736-88.

2. Ettehad D, Emdin CA, Kiran A, et al. Blood pressure lowering for prevention of cardiovascular disease and death: a systematic review and meta-analysis. The Lancet 2016;387:957-67.

3. Katsanos AH, Filippatou A, Manios E, et al. Blood Pressure Reduction and Secondary Stroke Prevention: A Systematic Review and Metaregression Analysis of Randomized Clinical Trials. Hypertension 2017;69:171-9.

4. Whelton PK, Carey RM, Aronow WS, et al. 2017 ACC/ AHA/AAPA/ABC/ACPM/AGS/APhA/ASH/ASPC/ NMA/PCNA Guideline for the Prevention, Detection, Evaluation, and Management of High Blood Pressure in Adults: A Report of the American College of Cardiology/ American Heart Association Task Force on Clinical Practice Guidelines. Hypertension 2018;71:e13-115.

5. Xie XX, Liu P, Wan FY, et al. Blood pressure lowering and stroke events in type 2 diabetes: A network metaanalysis of randomized controlled trials. Int J Cardiol 2016;208:141-6.

6. Jeffers BW, Robbins J, Bhambri R. Efficacy of Calcium Channel Blockers Versus Other Classes of Antihypertensive Medication in the Treatment of Hypertensive Patients With Previous Stroke and/or Coronary Artery Disease: A Systematic Review and MetaAnalysis. Am J Ther 2017;24:e68-80.

7. Mukete BN, Cassidy M, Ferdinand KC, et al. Long-Term Anti-Hypertensive Therapy and Stroke Prevention: A Meta-Analysis. Am J Cardiovasc Drugs 2015;15:243-57.

8. Chen GJ, Yang MS. The effects of calcium channel blockers in the prevention of stroke in adults with hypertension: a meta-analysis of data from 273,543 participants in 31 randomized controlled trials. PLoS One 2013;8:e57854.

9. Lu G AA. Combination of direct and indirect evidence in mixed treatment comparisons. Stat Med 2004;23:3105-24.

10. G S. Indirect and mixed-treatment comparison, network, ormultiple-treatments meta-analysis: many names, many benefts, many concerns for the next generation evidence synthesis tool. Res Synth Methods 2013;3:80-97.

11. Moher D, Liberati A, Tetzlaff J, et al. Preferred reporting items for systematic reviews and meta-analyses: the PRISMA statement. PLoS Med 2009;6:e1000097.

12. Hutton B, Salanti G, Caldwell DM, et al. The PRISMA extension statement for reporting of systematic reviews incorporating network meta-analyses of health care interventions: checklist and explanations. Ann Intern Med 2015;162:777-84.

13. Law MR, Morris JK, Wald NJ. Use of blood pressure lowering drugs in the prevention of cardiovascular disease: meta-analysis of 147 randomised trials in the context of expectations from prospective epidemiological studies. BMJ 2009;338:b1665.

14. Higgins J, Green SE. Cochrane Handbook for Systematic Reviews of Interventions Version 5.1.0. Available online: https://handbook-5-1.cochrane.org/

15. Higgins JP, Thompson SG, Deeks JJ, et al. Measuring inconsistency in meta-analyses. BMJ 2003;327:557-60.

16. Egger M, Davey Smith G, Schneider M, et al. Bias in meta-analysis detected by a simple, graphical test. BMJ 1997;315:629-34.

17. Dias S, Welton NJ, Sutton AJ, et al. NICE DSU technical support document 2: a generalised linear modelling framework for pairwise and network meta-analysis of randomized controlled trials. London: National Institute for Health and Care Excellence (NICE), 2010.

18. Lambert PC, Sutton AJ, Burton PR, et al. How vague is vague? A simulation study of the impact of the use of vague prior distributions in MCMC using WinBUGS. Stat Med 2005;24:2401-28.

19. Dias S, Welton NJ, Sutton AJ, et al. Evidence synthesis for decision making 4: inconsistency in networks of evidence based on randomized controlled trials. Med Decis Making 2013;33:641-56.

20. Biondi-Zoccai G, Abbate A, Benedetto U, et al. Network meta-analysis for evidence synthesis: what is it and why is it posed to dominate cardiovascular decision making? Int J Cardiol 2015;182:309-14.

21. Gelman A, Rubin D. Inference from Iterative Simulation Using Multiple Sequences. Stat Sci 1992;7:457-72. 


\section{Page 6 of 6}

22. Dias S, Welton NJ, Caldwell DM, et al. Checking consistency in mixed treatment comparison meta-analysis. Stat Med 2010;29:932-44.

23. Lu G, Ades AE. Assessing evidence inconsistency in mixed treatment comparisons. J Am Stat Assoc 2006;101:447-59.

24. Higgins JP, Jackson D, Barrett JK, et al. Consistency and inconsistency in network meta-analysis: concepts and models for multi-arm studies. Res Synth Methods

Cite this article as: Zhong XL, Dong $\mathrm{Y}, \mathrm{Xu} \mathrm{W}$, Sun L, Wang HF, Li HQ, Huang YY, Tan L, Dong Q, Yu JT. Blood pressure lowering and stroke prevention: a systematic review and network meta-analysis protocol. Ann Transl Med 2019;7(18):489. doi: 10.21037/atm.2019.08.99

\section{Zhong et al. Blood pressure lowering and stroke prevention}

2012;3:98-110.

25. Salanti G, Ades AE, Ioannidis JP. Graphical methods and numerical summaries for presenting results from multipletreatment meta-analysis: an overview and tutorial. J Clin Epidemiol 2011;64:163-71.

26. Chaimani A, Higgins JP, Mavridis D, et al. Graphical tools for network meta-analysis in STATA. PLoS One 2013;8:e76654. 


\section{Appendix 1: Electronic search strategies for PubMed}

HYPERTENSION/dt [dt=Drug Therapy] BLOOD PRESSURE/de [de=Drug Effects] ANTIHYPERTENSIVE AGENTS/tu [tu=Therapeutic Use]

*VASODILATOR AGENTS/(vasodilator* AND agent*).ti ambrisentan.ti

bosentan.ti

*DIAZOXIDE/diazoxide.ti

*HYDRALAZINE/hydralazine.ti

*ILOPROST/iloprost.ti

*MINOXIDIL/minoxidil.ti

sildenafil.ti

*NITROPRUSSIDE/nitroprusside.ti

tadalafil.ti

${ }^{*}$ METHYLDOPA/methyldopa.ti

${ }^{*}$ CLONIDINE/clonidine.ti

moxonidine.ti

*GUANETHIDINE/guanethidine.ti

*ADRENERGIC ALPHA-

ANTAGONISTS/*DOXAZOSIN/doxazosin.ti

*INDORAMIN/indoramin.ti

*PRAZOSIN/prazosin.ti

terazosin.ti

*PHENOXYBENZAMINE/phenoxybenzamine.ti

*PHENTOLAMINE/phentolamine.ti

*ADRENERGIC BETA-

ANTAGONISTS/*ATENOLOL/atenolol.ti

*METOPROLOL/metoprolol.ti

*PINDOLOL/pindolol.ti

*TIMOLOL/timolol.ti

*OXPRENOLOL/oxprenolol.ti

nebivolol.ti

*NADOLOL/nadolol.ti

*LABETALOL/labetalol.ti

${ }^{*}$ CELIPROLOL/celiprolol.ti

carvedilol.ti

*BISOPROLOL/bisoprolol.ti

*ACEBUTOLOL/acebutolol.ti

*PROPRANOLOL/propranolol.ti

*SODIUM CHLORIDE SYMPORTERINHIBITORS/

(diuretic* ANDthiazide*).ti

*HYDROCHLOROTHIAZIDE/hydrochlorothiazide.ti

*TRICHLORMETHIAZIDE/trichlormethiazide.ti

*SPIRONOLACTONE/spironolactone.ti

${ }^{*}$ CHLORTHALIDONE/chlorthalidone.ti
*INDAPAMIDE/indapamide.ti

*ANGIOTENSIN-CONVERTING

ENZYMEINHIBITORS/(ace AND inhibitor*).ti

${ }^{*}$ CAPTOPRIL/captopril.ti

*CILAZAPRIL/cilazapril.ti

*ENALAPRIL/enalapril.ti

*FOSINOPRIL/fosinopril.ti

imidapril.ti

${ }^{*}$ LISINOPRIL/lisinopril.ti

moexipril.ti

*PERINDOPRIL/perindopril.ti

quinapril.ti

*RAMIPRIL/ramipril.ti

trandolapril.ti

*ANGIOTENSIN II TYPE 1 RECEPTOR BLOCKERS/

azilsartan.ti

candesartan.ti

eprosartan.ti

irbesartan.ti

*LOSARTAN/losartan.ti

olmesartan.ti

telmisartan.ti

valsartan.ti

(renin AND inhibitor*).ti

aliskiren.ti

*CALCIUM CHANNEL BLOCKERS/*AMLODIPINE/

amlodipine.ti

${ }^{*}$ DILTIAZEM/diltiazem.ti

${ }^{*}$ FELODIPINE/felodipine.ti

*ISRADIPINE/isradipine.ti

lacidipine.ti

lercanidipine.ti

*NICARDIPINE/nicardipine.ti

*NIFEDIPINE/nifedipine.ti

*NISOLDIPINE/nisoldipine.ti

*VERAPAMIL/verapamil.ti

*NITRENDIPINE/nitrendipine.ti

Or/1-75

Cerebrovascular Disorders[all fields]

stroke[all fields]

Brain infarction[all fields]

Cerebral infarction[all fields]

Brain ischemia[all fields]

Cerebral hemorrhage[all fields]

Intracranial Hemorrhages[all fields]

Mortality[all fields]

Or/77-84

76 and 85 